\title{
Représentation statistique des alternances pluie-mon pluie à partir des séries pluviographiques Applications à Montpellier-Bel Air (1920-1971) et Paris-Montsouris (1949-1978)
}

\author{
Statistical representation of the alternation \\ of dry and rainy spells using rainfall series \\ Applications at Montpellier-Bel Air (1920-1971) \\ and Paris-Montsouris (1949-1978)
}

\author{
J. M. Masson \\ Université des Sciences et Techniques \\ du Languedoc, Montpellier
}

\section{Introduction}

Parmi les moyens mis en oeuvre pour mieux appréhender les risques liés à la grande variabilité des écoulements de surface, la modélisation figure actuellement en bonne place. Ainsi, par exemple, des hydrogrammes de crue pourront être simulés à l'exutoire d'un bassin versant, à partir d'une longue série observée ou synthétique de précipitations. Ces hydrogrammes permettent une estimation des conséquences des crues meilleure que la seule connaissance d'une valeur particulière (comme le débit de pointe) qui est plus facile à obtenir par d'autres méthodes.

Ces techniques de modélisation sont particulièrement adaptées aux petits bassins urbanisés, quand le temps de réponse est court (quelques dizaines de minutes) et quand les inconnues liées à la capacité d'absorption des sols et à l'hétérogénéité spatiale de la pluie sont peu importantes. Dans ces conditions, la façon dont se succèdent les averses joue un grand rôle, et on peut toujours craindre que la pluie de projet, construite autour d'une caractéristique pluviométrique de fréquence donnée, ne soit qu'un représentant d'une famille de pluie capables de conduire à des crues similaires.

L'intérêt de la connaissance de la structure ponctuelle de la pluie à un pas de temps fin (quelques minutes) pour aménager l'évacuation des eaux de ruissellement des petits bassins n'est d'ailleurs pas limité aux régions urbaines et s'étend aussi aux zones rurales dans la mesure où le comportement du sol est bien maîtrisé. Nous sommes d'ailleurs persuadés que le comportement des sols vis-à-vis de l'érosion et de l'infiltration, à la parcelle et sous des pluies naturelles, s'expliquerait beaucoup mieux par la prise en compte de la structure de la pluie à un pas de temps fin.

Le travail présenté ici correspond à la première des deux étapes prévues pour la modélisation stochastique des séries pluviographiques. Les hypothèses du modèle statistique décrit ont principalement été testées sur la série de Montpellier-Bel Air et transposées, après quelques vérifications fondamentales, sur la série de ParisMontsouris.

Dans une étape ultérieure, nous envisageons de modéliser l'intérieur des durées de pluie continue.

\section{Schématisation du processus}

Les séries pluviographiques sont discrétisées au millième de jour et au dixième de millimètre. A tout intervalle de temps de la discrétisation, est attribué un état selon la valeur de l'intensité de la pluie : supérieure ou égale à $0,1 \mathrm{~mm} /$ heure, c'est l'état pluvieux; sinon, c'est l'état sec. La série pluviographique est donc schématisée par une suite alternée de durées sèches $D s$ et de durées pluvieuses $D h$, ces dernières étant caractérisées aussi par une hauteur de pluie continue $H$ (Fig. 1). Pour tenir compte des variations saisonnières. Nous avons étudié séparément chaque période de l'année correspondant à un mois civil.

Analyse de la série chronologique des variables à Montpellier-Bel Air

Elle a pour but essentiel de détecter une éventuelle dépendance entre les réalisations successives des variables.

\section{Suite des hauteurs de pluie continue}

Une première mesure de dépendance est donnée par les coefficients linéaires d'auto-corrélation. Comme Yevjevich [1], avec des effectifs supérieurs à 30 , nous avons utilisé la transformation $Z$ de Fisher pour tester l'hypothèse nulle : $\rho 1=0$, contre l'hypothèse alter- 


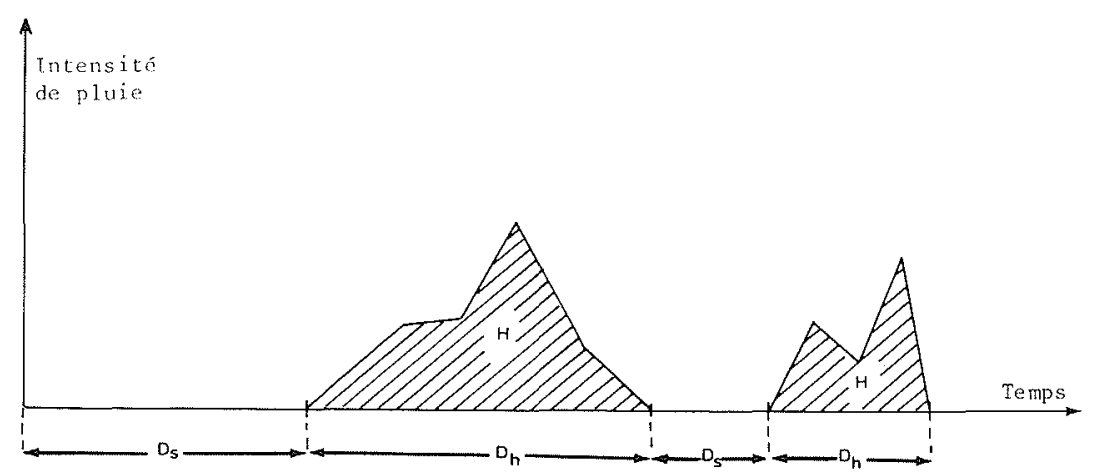

Figure 1 - Schématisation de la série pluviographique.

native : $\rho 1 \neq 0(\rho 1=$ coefficient linéaire d'autocorrélation avec retard de 1 dans la population). En considérant 2 sous-séries de 26 ans et au seuil $5 \%$, l'hypothèse nulle est rejetée pour 2 saisons sur 12 dans un cas et pour 5 saisons pour l' autre cas.

Comme les tests sur $\rho 1$ font l'hypothèse de la normalité des distributions et que nous sommes très loin de ces conditions, des tests non paramétriques ont été appliqués.

A partir d'un seuil de hauteur Ho déterminant deux étaț : inférieur ou égal à $H o$ et supérieur à $H o$, il est possible d'utiliser une distance $\chi^{2}$ pour tester l'hypothèse nulle d'indépendance dans la succession des états, contre l'hypothèse alternative d'une dépendance d'ordre 1 [2]. Avec $H o=0,4 \mathrm{~mm}$, deux sous-séries de 26 ans et un seuil de $5 \%$, l'indépendance est acceptée 9 fois sur 12 dans un cas et 10 fois dans l'autre. Au seuil $1 \%$, elle est toujours acceptée.

D'autres tests non paramétriques décrits par Yevjevich [1] d'après les travaux de Feller [3] analysent les séquences chronologiques supérieures ou inférieures à une valeur de variable $H(p) . H(p)$ est le quantile de la variable $I$ lié à la probabilité de non dépassement $p$; $(p=F[H(p)])$.

Pour différentes valeurs de $p$. on calcule les longueurs moyennes des séquences et on regarde si elles sont dans l'intervalle de confiance qu'on serait en droit d'attendre avec l'hypothèse d'indépendance.

Les résultats de ces derniers tests, ainsi que ceux des tests précédents, nous éclairent en définitive assez peu sur la dépendance chronologique des hauteurs. Bien que la proportion d'acceptations soit toujours relativement insuffisante pour admettre sans restriction l'hypothèse d'indépendance, les contradictions ne manquent pas. Par exemple. pour un même test, entre les deux sousséries de 26 ans, les rejets d'indépendance ne concernent pas les mêmes saisons. Ou encore les mois à fort coefficient d'autocorrélation ont des tests non paramétriques qui acceptent toujours l'indépendance ou vice-versa.

Dans ces conditions, l'indépendance des hauteurs sucessives est acceptée et les résultats obtenus en simulation seront juges du bien fondé de cette hypothèse.

\section{Suite des durées sèches}

Leur distribution bimodale [4] justifie une distinction en deux états : courts et longs, sur lesquels on peut effectuer un test $\chi^{2}$ d'indépendance, lequel est accepté 9 mois sur 12 au seuil $10 \%$ sur l'ensemble de la série.

Les tests non paramétriques des longueurs moyennes des séquences inférieures ou supérieures à un quantile $D s(p)$ donnent des résultats tantôt favorables (JanvierFévrier), tantôt défavorables (Juillet) mais pour des quantiles correspondant à des probabilités extrêmes alors que la représentativité des tests est discutable.

\section{Suite des couples successifs : Durée sèches-Durées pluvieuses}

Du fait de l'alternance des états, on trouve dans la série chronologique des paires successives $(D h, D s)$ ou $(D s, D h)$. Pour chacun des états secs ou pluvieux, on peut distinguer en plus deux classes de durées selon qu'elles sont inférieures ou égales ( $i=1)$ ou supérieures $(i=2)$ à un quantile lié à la fréquence marginale de non dépassement $p$. En cas d'indépendance, la fréquence d'un couple comportant un état de la classe $i$ par rapport a tous les couples comportant cet état, est toujours très voisine de la fréquence marginale. Si on appelle $N(D s i, D h i)$ le nombre de couples caractérisés par les classes d'états entre parenthèses, on aura par exemple :

$N(D s 1, D h 1) /[N(D s 1, D h 1)+N(D s 1, D h 2)] \simeq p$

Pour toutes les vérifications que nous avons effectuées, l'indépendance des tableaux de contingence $2 \times 2$ ainsi formés est acceptée. On trouve cependant sensiblement plus de durées sèches longues après les durées pluvieuses courtes qu'à la suite des durées pluvieuses longues. Mais encore une fois, nous sommes dans une situation limite pour décider de la dépendance ou de l'indépendance et nous devons compter sur les résultats obtenus en simulation pour trancher.

\section{Couples concomitants : Durée pluvieuse - Hauteur de pluie continue}

Ces deux variables sont dépendantes. Plus la durée pluvieuse est longue, plus la hauteur de pluie continue tend à être importante. On constate qu'après transfor- 


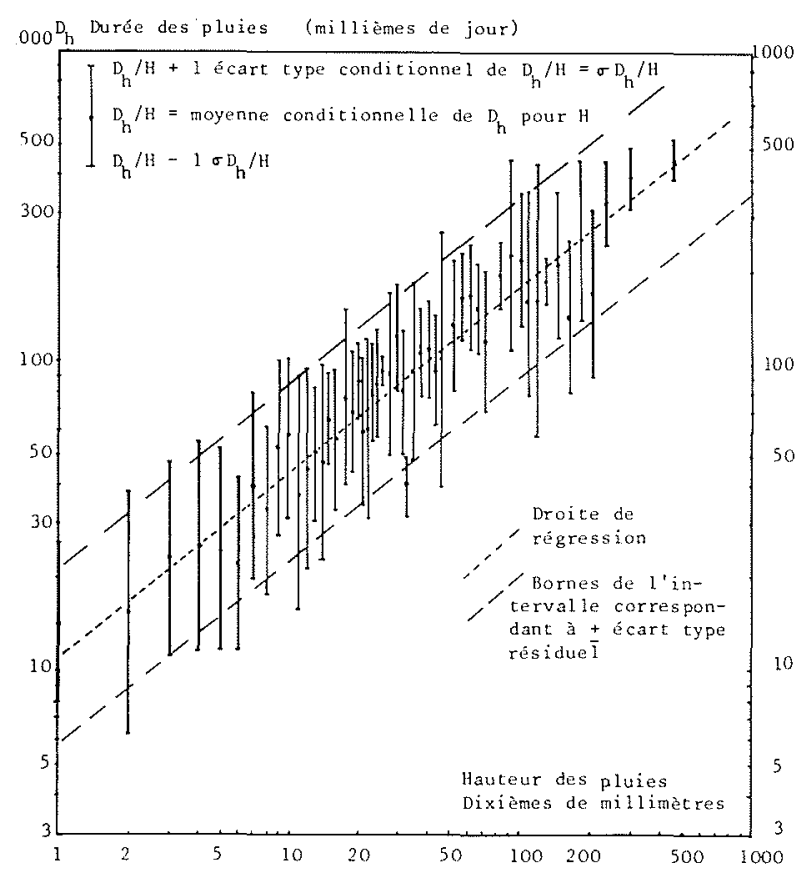

Figure 2 - Montpellier-Bel Air. Mois de Mars, Série 1946-1971. Liaison durée des pluies continues - Hauteur.

mation logarithmique, la relation $D h-H$ peut être représentée correctement par une droite et que l'homoscédasticité est approximativement respectée (Fig. 2). La distribution des résidus autour des moyennes conditionnelles n'a pas été spécialement étudiée et dans un premier temps nous avons adopté une loi normale.

\section{Distribution de probabilité des hauteurs de pluies continues à Montpellier-Bel Air}

Dans un précédent article [4], nous avons montré que les faibles hauteurs sont trop fréquentes pour qu'une loi simple puisse représenter l'ensemble de la distribution.
En portant les fréquences de dépassement en fonction des hauteurs sur un papier semi-logarithmique (Fig. 3), on voit que les 4 premières valeurs (1-2-3 et 4 dixièmes de millimètre) sont toujours alignées sur une droite passant par les point $(0,1)$. On peut donc décrire leur distribution par une loi exponentielle : $\mathrm{Si} p 4$ est la proportion des hauteurs $H \leqslant 4$ dixièmes de millimètre. la loi de $H \leqslant 4$ est décrite par :

$$
\begin{gathered}
\text { Prob }(H<h)=F(h)=1-e^{\alpha h} \\
\text { avec } \quad \operatorname{Ln}(1-p 4) / 4 \\
F(h) \leqslant p 4
\end{gathered}
$$

La proportion $p 4$ est peu soumise aux fluctuations saisonnières. Sur l'ensemble de la période d'observation et les 12 saisons distinguées, ses valeurs sont comprises entre 0,40 et 0,46 .

En ce qui concerne les hauteurs supérieures à 4 dixièmes de millimètre. Leur distribution est caractérisée par un coefficient de variation voisin de deux et un coefficient de dissymétrie compris entre 3 et 5 . La loi de probabilité d'expression simple qui donne les meilleurs résultats est la loi exponentielle généralisée ou loi de Weibull (Fig. 4) telle que :

$$
\operatorname{Prob}(H<h / H>4)=1-e^{-A(h-4,5)^{B}}
$$

Les tests $\chi^{2}$ d'ajustement au seuil $5 \%$ conduisent à 2 rejets sur 12 pour une sous-série (Août et Décembre) et à 4 rejets sur 12 pour l'autre (Février, Août, Octobre et Décembre). Cependant, pour une même saison, d'une sous-série à l'autre, on observe des différences importantes que la loi de Weibull a des difficultés à reproduire à partir des paramètres ajustés sur l'ensemble de la période d'observation.

Après de nombreux essais infructueux, nous avons été amenés à proposer une loi spéciale qui traduit le fait qu'en papier semi-logarithmique, les fréquences empiriques de dépassement dessinent une courbe d'allure

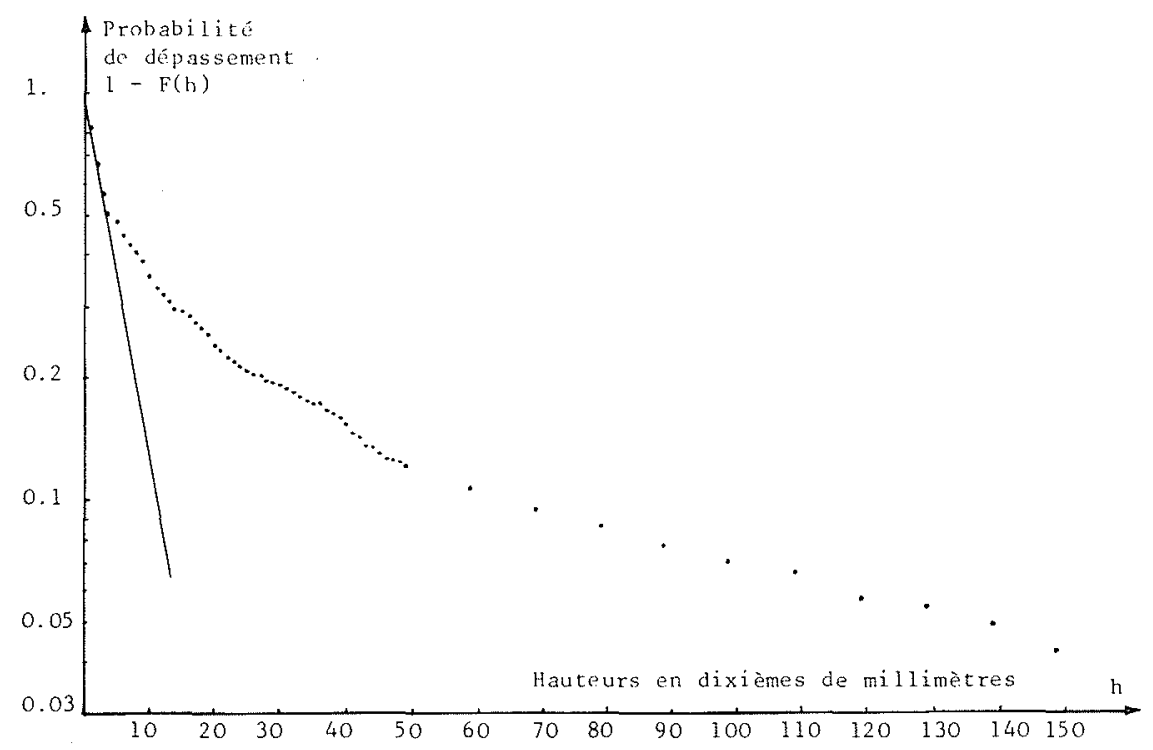

Figure 3 - Montpellier-Bel Air. Mois de Mai 1948-1971. Distribution des hauteurs de pluie continue. 


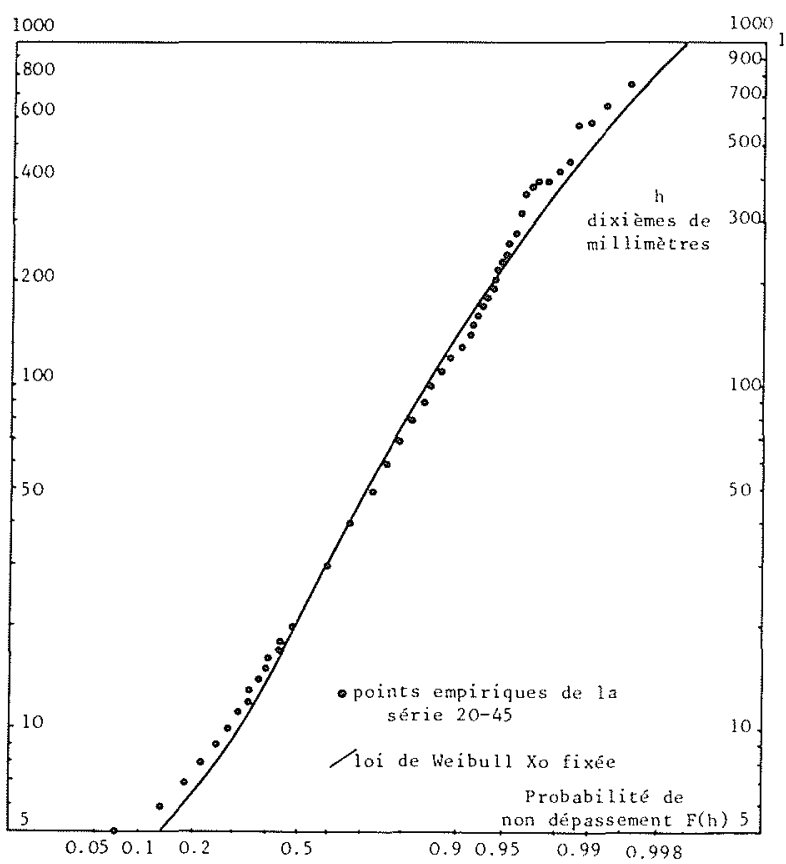

Figure 4 - Montpellier-Bel Air. Mois de Mars 1920-1945. Distribution des hauteurs de pluie continue $(h>0.4 \mathrm{~mm})$.

parabolique (Fig. 5). On peut ajuster facilement par les moindres carrés :

$$
\begin{gathered}
h^{\prime}=a\left[\operatorname{Ln}\left(1-F\left(h^{\prime}\right)\right)\right]^{2}+b\left[\operatorname{Ln}\left(1-F\left(h^{\prime}\right)\right)\right] \\
h^{\prime}=h-4
\end{gathered}
$$

ce qui conduit à l'expression suivante pour la fonction de répartition :

$$
\begin{gathered}
F\left(h^{\prime}\right)=1-e^{\left(-b-\sqrt{\left.b^{2}+a h^{\prime}\right) / 2 a}\right.} \\
b<0 \quad a>0
\end{gathered}
$$

Les tests $\chi^{2}$ d'ajustement au seuil $5 \%$, effectués dans les mêmes conditions que pour la loi de Weibull, ne donnent aucun rejet sur une sous-série et un seul rejet pour l'autre sous-série.
Cette loi "Parabole inverse" se montrant efficace, nous avons calculé l'expression des deux premiers moments par rapport à l'origine en fonction des paramètres. On a :

$$
\begin{aligned}
& m_{1}\left(h^{\prime}\right)=e^{-b / a}\left(\frac{b^{2}}{4 a}+b+2 a\right)-\frac{b^{2}}{4 a} \\
& m_{2}\left(h^{\prime}\right)=e^{-b / a} \\
& \left(-\frac{b^{4}}{16 a^{2}}+2 b^{2}+12 a b+24 a^{2}\right)+\frac{b^{4}}{16 a^{2}}
\end{aligned}
$$

Une méthode numérique itérative permet de trouver rapidement les valeurs de $a$ et $b$ à partir des deux premiers moments de la variable $h^{\prime}$. Pour trois saisons, quand

$$
\sqrt{m_{2}\left(h^{\prime}\right) / 24}>m_{1}\left(h^{\prime}\right) / 2
$$

on a une solution avec $b=0$; la loi s'écrit alors :

$$
F\left(h^{\prime}\right)=1-e \sqrt{\frac{h^{\prime}}{a}}
$$

Ce type de loi permet de bien retrouver la variabilité des sous-séries avec un seul jeu de paramètres.

\section{Distribution de probabilité des durées sèches à Montpellier-Bel Air}

Dans un article précédent [4], nous avons présenté ces distributions bimodales. Nous leur avons ajusté un mélange de deux distributions log-normales où la variable $y=\log D s$ est caractérisée par la distribution de probabilité :

$$
f(y)=p f_{1}(y)+(1-p) f_{2}(y)
$$

avec

$$
f_{i}(y)=\frac{1}{\sqrt{2 \pi \sigma_{i}}} e^{-1 / 2\left(\frac{y-\mu_{i}}{\sigma_{i}}\right)^{2}}(i=1,2)
$$

densité de probabilité d'une loi de Gauss.

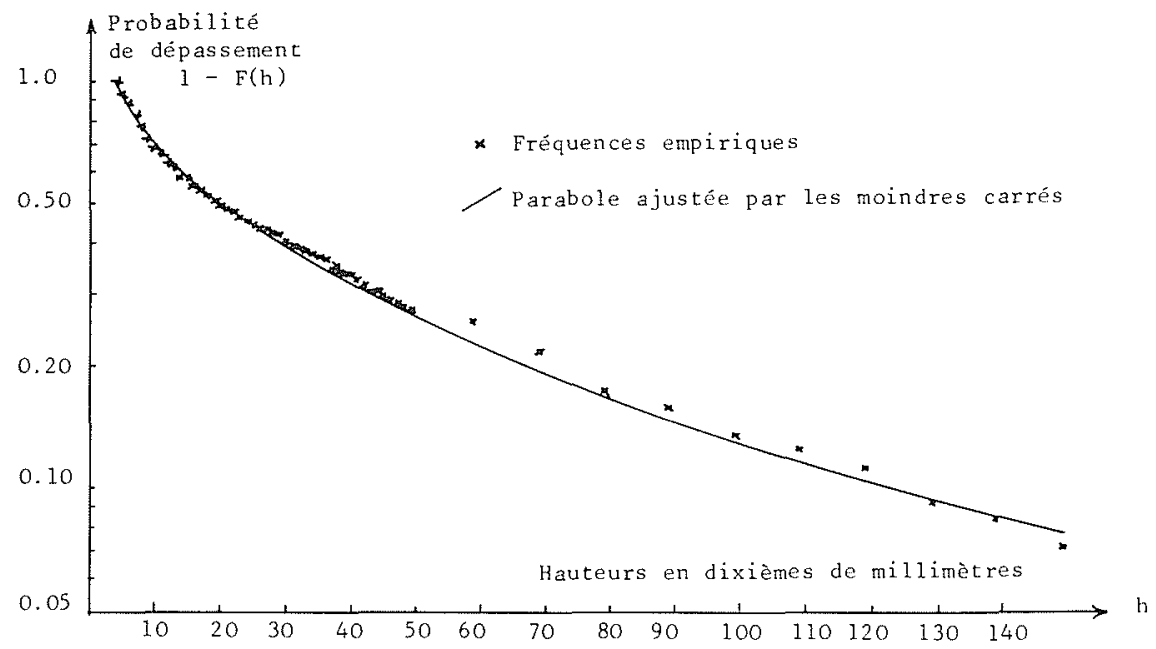

Figure 5 - Montpellier-Bel Air, Mois de Mai 1920-1945. Distribution des hauteurs des pluies continues $(h>0.4 \mathrm{~mm})$. 
Les paramètres : $p, \mu_{1}, \sigma_{1}, \mu_{2}, \sigma_{2}$ peuvent être estimés par la méthode des moments. Si $m_{j}(j=1,2,3,4,5)$ sont les moments d'ordre $j$ par rapport à l'origine calculés sur l'échantillon, la solution du système suivant, comportant 5 équations non linéaires à 5 inconnues, fournit une estimation des paramètres cherchés.

$$
\begin{aligned}
& m_{1}=p \mu_{1}+(1-p) \mu_{2} \\
& m_{2}=p\left(\sigma_{1}^{2}+\mu_{1}^{2}\right)+(1-p)\left(\sigma_{2}^{2}+\mu_{2}^{2}\right) \\
& m_{3}=p \mu_{1}\left(3 \sigma_{1}^{2}+\mu_{1}^{2}\right)+(1-p) \mu_{2}\left(3 \sigma_{2}^{2}+\mu_{2}^{2}\right) \quad(11) \\
& m_{4}=p\left[3 \sigma_{1}^{2}\left(2 \mu_{1}^{2}+\sigma_{1}^{2}\right)+\mu_{1}^{4}\right]+ \\
& \quad+(1-p)\left[3 \sigma_{2}^{2}\left(2 \mu_{2}^{2}+\sigma_{2}^{2}\right)+\mu_{2}^{4}\right] \\
& \left.m_{5}=p\left[5 \mu_{1} \sigma_{1}^{2}\left(3 \sigma_{1}^{2}+2 \mu_{1}^{2}\right)+\mu_{1}^{5}\right)\right]+ \\
& \quad+(1-p)\left[5 \mu_{2} \sigma_{2}^{2}\left(3 \sigma_{2}^{2}+2 \mu_{2}^{2}\right)+\mu_{2}^{5}\right]
\end{aligned}
$$

Une solution analytique du système a été proposée par Cohen [5]. Elle aboutit à une solution particulière d'un polynôme de degré neuf. Nous avons préféré utiliser une solution numérique proposée par Hawkins [6].

Les tests $\chi^{2}$ d'ajustement au seuil $5 \%$ conduisent à 4 rejets sur 12 saisons pour une sous-série, mais à des rejets systématiques pour l'autre sous-série. Nous avons attribué cette différence à une dérive de l'appareillage modifiant les conditions d'enregistrement au cours du temps.

\section{Caractères particuliers de la série de Paris- Montsouris}

Les éléments de base, valables sur la série de Montpellier, sont conservés : indépendance des variables successives, distribution des hauteurs de pluie continue décrite par deux lois de part et d'autre d'un seuil $H o$, liaison linéaire en logarithme pour les couples $D h-H$, mélange de deux lois log-normales pour décrire la distribution de $D s$.

Les principales différences concernent :

- le seuil Ho des hauteurs de pluie continue de part et d'autre duquel on change de loi de probabilité : 2 dixièmes de millimètre à Paris contre 4 dixièmes à Montpellier :

- la loi de probabilité qui décrit le mieux la distribution des hauteurs $H^{\prime} \stackrel{\text { q }}{=} H-H o$ n'est plus une loi "parabole inverse" mais une loi de Weibull ( 2 rejets sur 12 en Septembre et Octobre au seuil $5 \%$ contre 6 rejets).

\section{Simulations}

\section{Principes de la simulation}

Pour une saison, la succession des variables caractérisant le processus de pluie continue tel que nous l'avons schématisé, est simulée au moyen de 12 paramètres. Ce sont :

- les 4 paramètres décrivant la distribution des hauteurs de pluie continue c'est-à-dire : le seuil Ho, la probabilité
$(H \leqslant H o$ ) et soit les paramètres $A$ et $B$ d'une loi de Weibull, soit les paramètres $a$ et $b$ d'une loi "parabole inverse",

- les 3 paramètres de la régression entre les logarithmes de $D h$ et $H:$ le coefficient de régression $b(D h)$, l'ordonnée à l'origine $a(D h)$, l'écart type résiduel $\sigma D h$.

- les 5 paramètres du mélange de 2 lois log-normales décrivant la distribution des durées sèches $D s: p, \mu_{1}$, $\sigma_{1}, \mu_{2}, \sigma_{2}$.

La succession des opérations, pour une saison, est décrite par l'organigramme du Tableau I.

Pour simuler plusieurs années successives, le point de départ est fixé ; par exemple, le $1^{\text {er }}$ Janvier. Puis les durées sèches et pluvieuses successives sont cumulées au fur et à mesure de leur apparition, ce qui permet de repérer les changements de saison et de changer le jeu des paramètres.

Les valeurs des paramètres peuvent éventuellement être exprimées en fonction du jour de l'année par l'intermédiaire de fonctions périodiques. Les résultats sont alors plutôt moins bons pour un temps de calcul plus long.

\section{Valeurs des simulations}

Les résultats des simulations, suite alternée de durées sèches et de durées pluvieuses en millièmes de jour, ont été regroupés par tranches successives de 24 heures : un jour pendant lequel aucune pluie continue n'a été observée a été déclaré sec, sinon, pluvieux. Des jours secs successifs forment un épisode sec caractérisé par sa longueur. Des jours pluvieux successifs forment un épisode pluvieux caractérisé par sa longueur et une hauteur d'eau. Ces résultats sont identiques à ceux d'une série pluviométrique, à ceci près que les hauteurs de pluies journalières ne sont généralement pas connues parce qu'on ne sait pas répartir les pluies continues qui tombent à cheval sur deux jours.

$\mathrm{Ce}$ regroupement des pluies continues permet de vérifier que les principales caractéristiques d'un modèle de pluie journalière ajusté aux observations sont bien reproduites par la simulation. Ces caractéristiques sont : moyenne et écart type des durées des épisodes secs et pluvieux, moyenne et écart type des hauteurs des épisodes pluvieux. Nous avons aussi contrôlé les résultats au niveau des précipitations mensuelles (moyennes et écarts type).

Les comparaisons entre les caractéristiques observées et celles obtenues après simulation montrent une bonne concordance sauf pour deux d'entre elles :

- l'écart type des durées sèches, qui est systématiquement surestimé sur les séries simulées;

- l'écart type des hauteurs pluvieuses qui est systématiquement sous-estimé.

L'écart type des durées sèches est ramené à des valeurs acceptables si on borne supérieurement la loi des durées sèches longues à la plus forte valeur trouvée sur la série observée : 60 jours à Montpellier et 40 jours à Paris. Nous touchons du doigt le problème de la représentation de phénomènes physiquement bornés (les durées sèches) par des lois statistiques non bornées. 
Tableau I - Organigramme de la simulation des alternances pluie-non pluie

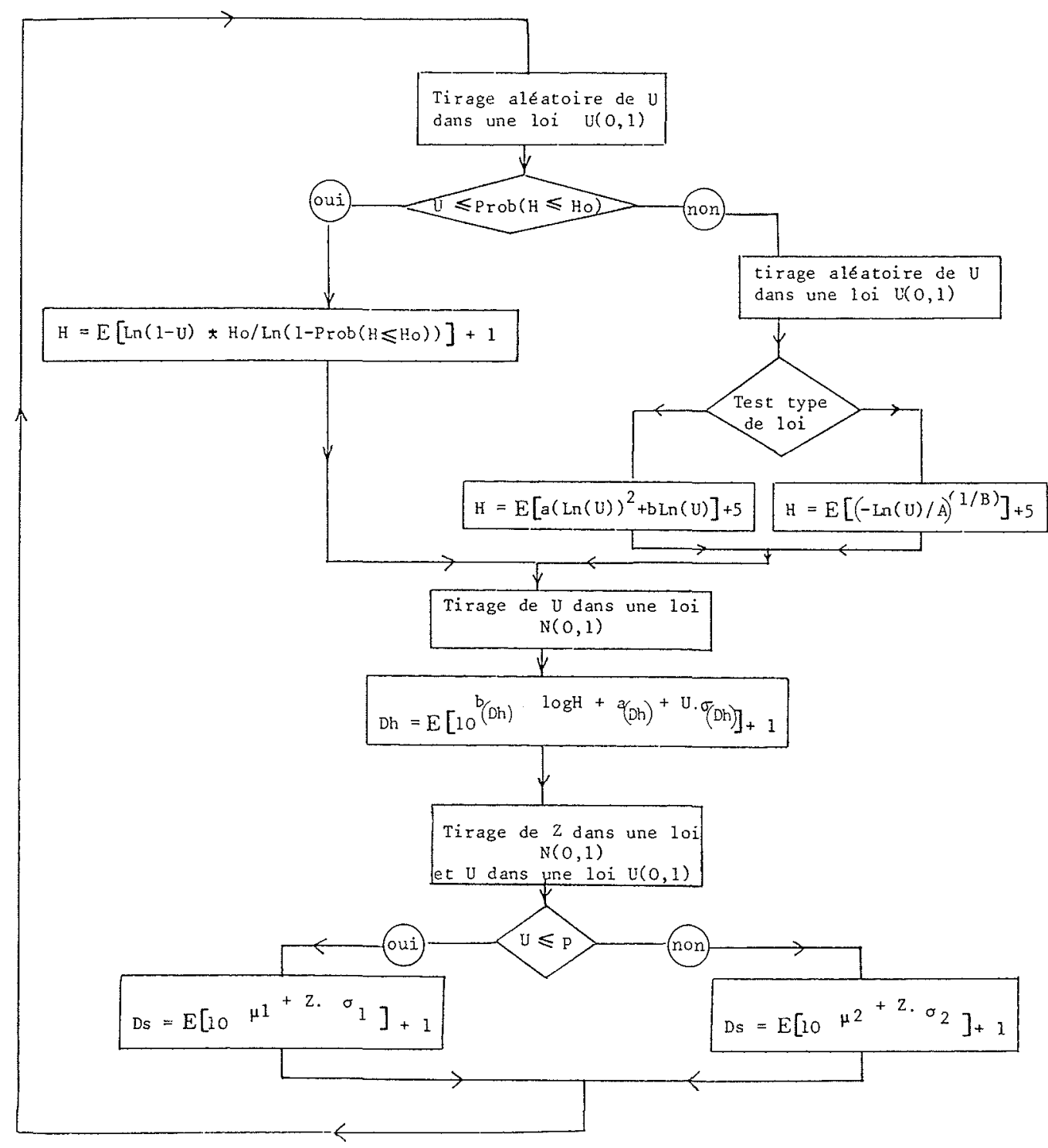

En ce qui concerne l'écart type des hauteurs des épisodes pluvieux, la cause de l'inadéquation est moins évidente. Elle pourrait provenir du fait que les valeurs successives de pluies continues séparées par des durées sèches courtes ont des caractéristiques voisines liées à la nature de la perturbation qui les provoque. Ainsi, à Montpellier, les pluies frontales d'origine atlantique apportées par les vents d'Ouest donnent des $H$ faibles associées à des $D h$ plutôt longues. Inversement, les pluies apportées par les vents du Sud ou les orages donnent des $H$ élevées associées à des $D h$ plutôt courtes.

On aurait donc des séquences de couples $H$-Dh de caractéristiques voisines jusqu'à ce qu'une durée sèche longue intervienne, ce dont nous n'avons pas tenu compte.

Pour retrouver la variabilité des hauteurs d'épisode, nous avons essayé :

- une auto-corrélation sur les résidus de la relation $D h-H$ fonction de la durée sèche précédente, ce qui a peu changé les résultats ;
- pour chacun des mois des années simulées :

- un tirage aléatoire de Prob $(H \leqslant H O)$ dans une loi uniforme de moyenne Prob $(H \leqslant H o)$ et de bornes Prob $\left(H \leqslant H_{0}\right) \pm 0.3$

- un tirage aléatoire dans une loi uniforme précisant la manière d'obtenir $H^{\prime}$ :

* dans la totalité de la loi ( $1 / 2$ des cas),

* dans la loi tronquée $H^{\prime} \leqslant H^{\prime}(0.5)$ (1/4 des cas),

* dans la loi tronquée $H^{\prime}>H^{\prime}(0.5)$ (1/4 des cas).

Ces procédures nous ont permis d'améliorer les résultats des simulations en ce qui concerne les écarts types des hauteurs des épisodes pluvieux journaliers (Fig. 6).

Il sera cependant souhaitable de reprendre l'analyse pour mettre en évidence cette typologie dans les séquences de pluies continues. 


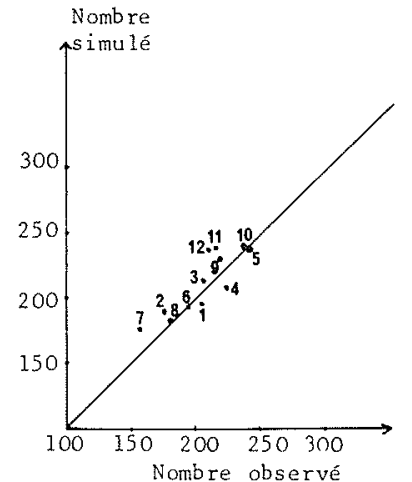

DUREE DES EPISODES PLUVIEUX
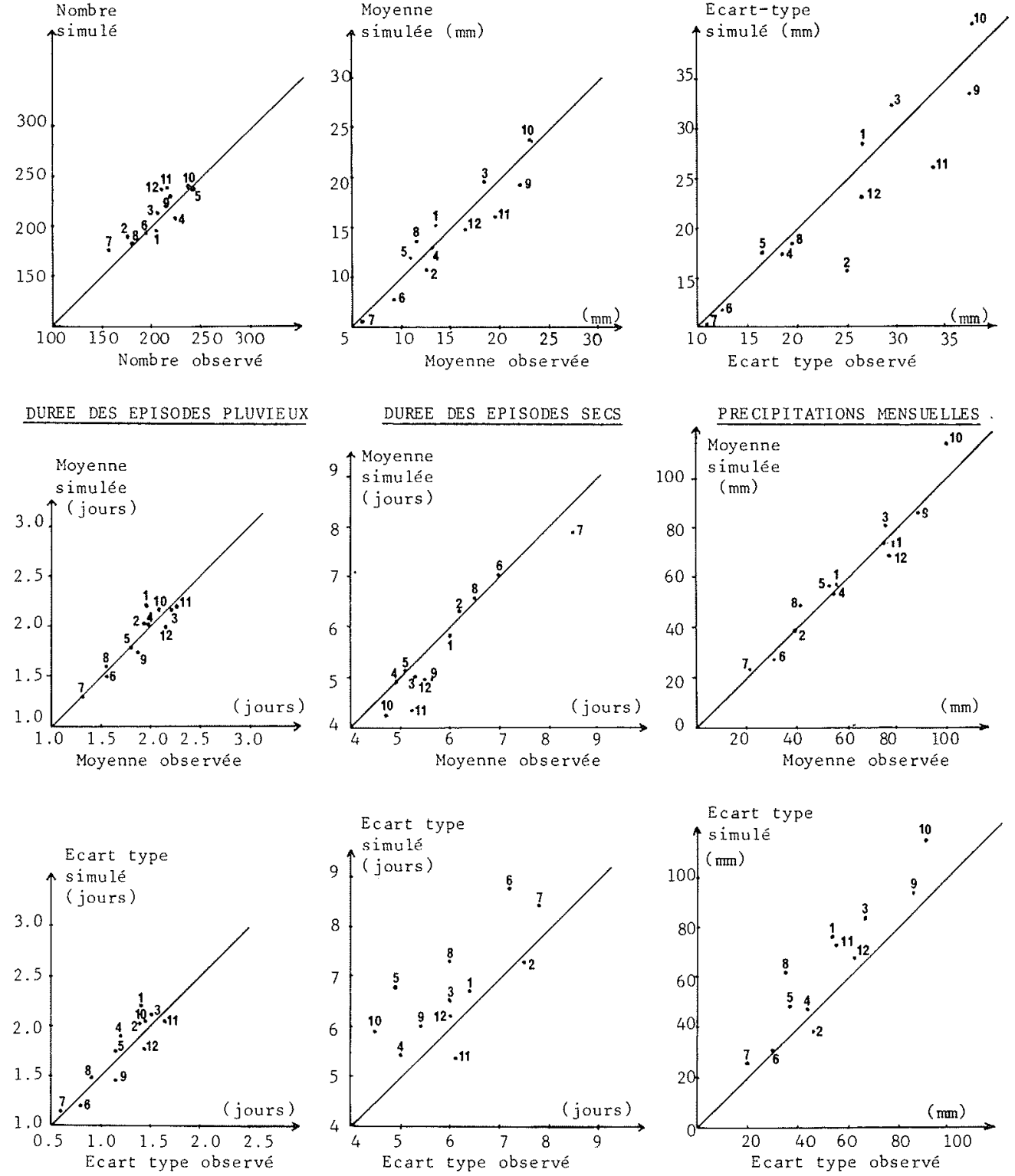

Figure 6 - Comparaison au niveau journalier de la série observée et d'une série simulée. Montpellier-Bel Air. 52 ans.

\section{Conclusion}

Nous remercions les Services du Ministère de l'Agriculture et la Météorologie Nationale qui nous ont autorisé à utiliser les données numérisées des deux grandes séries pluviographiques françaises pour faire cette étude.

La modélisation des précipitations à échelle fine ne sera effective que dans la mesure où la simulation permettra de retrouver la loi des précipitations journalières. L'objectif prochain concerne la répartition de la pluie à l'intérieur des durées de pluie continue, pour connaître ce qui est tombé un jour donné.

\section{Bibliographie}

[1] YEVJEVICH V. - Stochastic Processes in Hydrology. Water Ressources Publications, Fort-Collins, Colorado, $1972,276 \mathrm{p}$.

[2] MASSON J.M. - Persistance des états pluvieux en fonction de leur durée. Cahiers O.R.S.T.O.M., Série Hydrologie, 1977, Vol. XIV, n ${ }^{\circ} 2$, pp. 173-189.

[3] FELLER W. - An Introduction to Probability Theory and its Applications. John Wiley and Sons, $3^{\mathrm{e}}$ edition, 1968, Vol. 1, $509 \mathrm{p}$.

[4] MASSON J.M. - Analyse d'une longue série pluviographique. Montpellier-Bel Air 1920-1971. La Météorologie, 1980, VI Série, n 20-21, pp. 105-117.

[5] COHEN A.C. - Estimation in mixtures of two normal distributions. Technometrics, Février 1967, Vol. 9, $\mathrm{n}^{\circ} 1$, pp. 15-28.

[6] HAWKINS, R.H. - A note on multiple solutions to the mixed distribution problem. Technometrics, Novembre 1972, Vol. 14, n 4, pp. 973-976. 


\section{Discussion}

Président : M. M. ROCHE

Le Président. - Je remercie M. MASSON de son exposé. $\mathrm{Y}$ a-t-il des observations ou des commentaires sur cet exposé ?

M. DERI. - Je ferai une petite remarque. M. MASSON nous a indiqué qu'il y a une fonction de densité bimodaie. Cela indique s'il s'agit d'une fonction de répartition composée. Pour ces cas, je pourrais conseiller d'utiliser la fonction de $\beta$ qui se compose de deux fonctions $\gamma$, ce qui est très bon, d'après mes expériences.

M. MASSON. - Je vous remercie pour ce conseil; je ne manquerai pas d'essayer la loi $\beta$.

M. MARTIN. -- Pour simuler les précipitations, avez-vous utilisé des modèles de types markovien ou autres, permettant de rendre compte du phénomène de persistance d'un jour au jour suivant?

M. MASSON - Dans le modèle de simulation, les durées sèches et pluvieuses successives sont indépendantes; la seule dépendance lie la hauteur de pluie continue à sa durée.

M. MARTIN. - Plus précisément, lorsqu'on fait l'étude des séquences sèches on s'aperçoit que la probabilité de passage d'un jour sec au suivant dépend de la longueur de la séquence sèche en cours au moins pour un certain nombre de mois de l'année et pour un certain nombre de stations en France. Est-ce que le fait de ne pas prendre en compte ce phénomène de renfor- cement de la persistance en fonction de la longueur de la séquence n'est pas la cause des anomalies que vous observez au niveau de la reconstitution des écarts-type du nombre de jours secs par exemple?

M. MASSON. - Vous citez la persistance entre jours secs et pluvieux. Si la persistance est indéniable à cette échelle de temps, il ne semble pas en être ainsi quand on considère les durées de pluie continue. Dans ce cas, les durées pluvieuses et sèches successives qui sont souvent très courtes (quelques minutes) mais peuvent être aussi très longues (durées sèches de plusieurs dizaines de jours), semblent être indépendantes. Cependant les tests statistiques d'indépendance ne sont pas absolument décisifs et je vous accorde qu'il subsiste une incertitude à ce niveau.

M. OBLED. - Je voudrais savoir si les résultats que vous avez obtenus vous semblent tributaires du pas de dépouillement utilisé, ensuite du pas de simulation utilisé. Avez-vous l'impression que ce que l'on simule serait très différent si l'on prenait le pas de l'heure?

M. MASSON. - Le pas de simulation est celui des données utilisées pour calibrer le modèle. Je n'ai aucune idée des différences qu'on obtiendrait en utilisant un pas de simulation horaire.

Le Président. - S'il n'y a pas d'autres commentaires sur la communication de M. MASSON, je vais clore la discussion.

\section{Abstract \\ Statistical representation of the alternation of dry and rainy spells using rainfall séries - Applications at montpellier - Bel Air (1920-1971) and Paris Montsouris (1949-1978)}

This is an attempt at the statistical portrayal of a succession of showers at a station using the shortest possible time interval. Two long numerised rainfall series are used : Montpellier - Bel Air, provided by the Ministry of Agriculture and Paris Montsouris, supplied by the national meterorological agency. The discrete units used are a thousandth of a day for time and a tenth of a millimeter for water heights.

Successive precipitations are given schematically by an alternating series of dry spells (Ds) and rainy spells (Dh); the latter are also characterised by a continuous rainfall height $\mathrm{H}$. The statistical properties of this process are studied separately for each month of the year.

The marginal distribution of continuous rainfall heights $\mathrm{H}$ is characterised by a high frequency of heights lower than or equal to a threshold Ho $(0.4 \mathrm{~mm}$ at Montpellier $-0.2 \mathrm{~mm}$ in Paris). The frequency distribution of these low height is well described by an exponential law whose parameter is associated with the frequency of $\mathrm{H} \leqslant \mathrm{Ho}$. The distribution of values $\mathrm{H}^{\prime}$ hĭgher than the $\mathrm{Ho}\left[\mathrm{H}^{\prime}=\mathrm{H}-(\mathrm{Ho}+0.05)\right]$ is either protrayed by an exponental law after a parabolic transformation of the variable (Montpellier) or by a Weibull law (Paris).

The couples (Ds - H) are log-linearly dependent. The value of the correlation coefficient is between 0.6 and 0.8 according tothe season. Residual precipitation has a somewhat symmetrical distribution around the regression line, with a constant variance.

The frequency distribution of dry spells is bimodal. The first mode corresponds to short dry spells between successive showers, and the second to much longer spells of true good weather. This distribution is described by combining the two lognormal lanes in a proportion $p$.

The successive variables : spells and heights are all but independent. The tests carried out always lead to a slightly higher number of rejections than acceptances of the hypotheses of independence, for parametrical testing using the linear auto-correlation coefficient $\mathrm{pl}$, for non-parametrical testing, distinguishing between two states on either side of a numerical value, and for scrutiny of the average duration of chronological sequences which are lower or higher than a given quantile. However, when comparing the rejections under various tests of each half of a series, the same seasons are never systematically found. Independence has thus been accepted, subject to it providing accurate results during simulation tests.

Simulation offers no particular difficulty in the conditions stated above. To make a judgement of the results, rainfall heights have been added over 24 consecutive hours to obtain a series similar to a rainfall series in which successive rainy days have been cumulated. The observed and simulated series can then be compared at the level of the first two moments of the variables characterising a daily rainfall model : length in days of dry and rainy spells and the corresponding height of the latter. The comparison can also be made using monthly totals.

The first simulation shows reasonably satisfactory results, except for the standard deviations of length of dry spells (overestimated) and heights during rainy spells (under estimated).

The first anomaly is easily corrected by setting the maximum value observed as boundary to the dry spells (Ds). This touches on the problem of statistical portrayal of a physically bounded variable by a non-bounded law.

The second anomaly is linked to a typology of daily spells : some corresponding to cyclonic disturbances involve only light showers; by contrast, others associated with convective phenomena, involve only heavy showers.

It is found that a simulation distinguishing type of spell arbitrarily by drawing only from a small part of the law governing $H$ (but preserving the law governing $H$ over all the spells) improves the results.

The portrayal of a succession of showers at a station remains to be perfected by a typological study of spells and completed by a study of the succession of rain intensity within continuous spells of rainfall. 